\title{
Luminex-based quantification of Alzheimer's disease neuropathologic change in formalin-fixed post-mortem human brain tissue
}

\author{
C. Dirk Keene ${ }^{1} \cdot$ Angela M. Wilson $^{1} \cdot$ Mitchell D. Kilgore $\mathbb{C}^{1} \cdot$ Lauren T. Bruner $^{1} \cdot$ Nadia O. Postupna $^{1} \cdot$ Martin Darvas $^{1}$
}

Received: 20 July 2018 / Revised: 20 September 2018 / Accepted: 20 September 2018 / Published online: 20 December 2018

(c) United States \& Canadian Academy of Pathology 2018

\begin{abstract}
The vast majority of archived research and clinical pathological specimens are stored in the form of formalin fixed, paraffinembedded (FFPE) tissues, but, unlike fresh frozen tissue samples, highly quantitative measures in FFPE tissues are limited to immunohistochemical and immunofluorescence thresholding image analysis studies, cell counting, and ordinal ranking systems. This poses a significant obstacle for clinical investigations that aim to correlate diagnostic markers of neurodegenerative diseases like Alzheimer's disease (AD) with parameters like age, gender, drug exposures, genotype, disease stage, co-morbidities, or environmental factors. To overcome this limitation, we have developed Luminex-based techniques and protocols for the quantification of amyloid $\beta$ and hyperphosphorylated Tau in FFPE brain sections. We validated the Luminex assay in FFPE sections from prefrontal cortex, hippocampus, and neostriatum from 30 cases that underwent prior neuropathological diagnostic assessment of $\mathrm{AD}$ following the current NIA-AA recommendations for AD: 10 cases diagnosed as not or low, 10 cases as intermediate, and 10 cases as high $\mathrm{AD}$ neuropathologic change. Consistent with the neuropathologic assessment, Luminex assay detected high amounts of amyloid beta in the frontal cortex and striatum, and high amounts of hyperphosphorylated Tau in the frontal cortex and hippocampus, of cases with high AD neuropathologic change. This assay can be expanded to detect diverse antigenic targets of interest, as we show here with IBA1 and GFAP. This novel approach supports multiplexed highly quantitative, molecularly specific neuropathology measures to further explore mechanisms of neurodegeneration in AD.
\end{abstract}

\section{Introduction}

The pathognomonic lesions of Alzheimer's disease (AD) are neuritic plaques and neurofibrillary tangles (NFT) [1]. Amyloid $\beta$ peptide 42 (A $\beta 42)$ is a key component of neuritic plaques, and antibodies against $\mathrm{A} \beta 42$ are used in immunohistochemistry (IHC) procedures required for the neuropathologic diagnosis of AD. NFTs are primarily composed of hyperphosphorylated Tau (phospho-Tau

These authors contributed equally: C. Dirk Keene, Angela M. Wilson

Supplementary material The online version of this article (https:// doi.org/10.1038/s41374-018-0165-x) contains supplementary material, which is available to authorized users.

Martin Darvas

mdarvas@uw.edu

1 Department of Pathology, University of Washington, Seattle, WA 98104, USA
(pTau)), and antibodies against pTau epitopes are used in routine IHC procedures for neuropathologic assessment of AD. In fact, the current National Institute on Aging-Alzheimer's Association (NIA-AA) consensus criteria for the neuropathologic diagnosis of $\mathrm{AD}$ are based on histologic characterization of the anatomic distribution of amyloid plaques and NFTs [1,2]. These criteria are based on previously established criteria for $\mathrm{A} \beta$ plaque [3] and NFT distribution $[4,5]$, and cortical density of neuritic plaques [6]. These diagnostic criteria are not quantitative measures but rather ordered rankings, which severely limits their utility in research studies that aim to correlate pathological features of $\mathrm{AD}$ with parameters like age, gender, drug exposures, genotype, co-morbid processes, or environmental factors.

Because the vast majority of archived pathological tissues in research tissue banks and in clinical archives are maintained in the form of formalin-fixed paraffin-embedded (FFPE) tissue, approaches to quantify AD neuropathologic change in brain tissue are mostly restricted to IHC or 
histochemical methods. These approaches are inherently limited by issues relating to a variability of image thresholds, obtaining uniform optics across tissue, quenching endogenous signals, and field selection. Although unbiasedstereology-based protocols allow counting of antibodystained cells and other discrete structures [7], they do not usually provide a measure for quantifying antibody-binding events and are labor intensive.

Recently, automated image-processing protocols and tissue microarray techniques, similar to those employed in cancer studies, have been applied to neurodegenerative diseases to overcome some of these limitations $[8,9]$. For example, using computer-assisted image analyses, Boyle et al. have been able to associate IHC measures of amyloid and NFT pathology with ante-mortem cognitive parameters [10]. However, sensitivity to detect low abundance targets and optical correction factors including, but not limited to, background signal subtraction, can reduce sensitivity. A different approach to generate quantitative measures of $\mathrm{AD}$ neuropathologic change is a combination of histologic evaluation and ELISA performed on a standard glass slide ("Histelide"), which has been used on over 300 brain autopsies so far [11-14]. The Histelide method is molecularly specific, but is limited by high cost and inability to effectively multiplex the approach. Here, we report a fundamental transformation of quantitative FFPE-tissue-based techniques, by adding a Luminex-based approach to the analysis of FFPE tissue in neurodegenerative diseases that is cost-effective, sensitive, and can be multiplexed to limit tissue usage, while allowing cytoarchitectural correlation in immunohistochemically stained adjacent sections. Luminex technology combines magnetic-fluorescent bead-based immunoassays with advanced fluidics, optics, and signal processing to permit quantification of multiple antigens within a single sample [15]. This technology has been widely used in frozen-tissue extracts, as well as in serum and cerebrospinal fluid samples from patients with a wide range of diseases and disorders [16], including AD [17-20].

- Our laboratory has developed FFPE-compatible protocols that allow extraction of $\mathrm{A} \beta 42$, pTau, glial fibrillary acidic protein (GFAP), and ionized calcium-binding adapter molecule 1 (IBA1). In this manuscript we demonstrate the use of Luminex technology to quantify expression of these proteins in human FFPE sections from three brain regions included in the NIA-AA recommendations for neuropathologic assessment of $\mathrm{AD}$ [2]. Using this method, we verified increased amounts of A $\beta 42$ and pTau in brains with higher levels of AD neuropathologic change. The highly quantitative and multiplexed approach allows comparisons between levels of pathologic peptides and other markers, in the same tissue section, thus allowing for more precise assessment of correlations with risk factors and other parameters of interest. Using Luminex to quantify $A \beta 42$ and pTau in FFPE tissue offers several advantages that complement existing IHC-based analyses of neurodegenerative change in $\mathrm{AD}$, and provides new opportunities for molecular analyses of $\mathrm{AD}$ and other neurodegenerative diseases. Because of its inherent quantitative nature, high degree of sensitivity and specificity, and the ability to simultaneously analyze multiple (up to 48 if duplicate runs are used on a 96-well plate) samples on one Luminex plate, this assay is well suited for high-throughput analysis of A $\beta 42$ and pTau levels in FFPE autopsy brain tissue.

\section{Materials and methods}

\section{Human subjects}

This study was approved by the University of Washington (UW) Institutional Review Board. All cases were from the UW Alzheimer's Disease Research Center (ADRC) Neuropathology and Targeted Molecular Testing (NPTMT) Core. All study subjects were participants in the Adult Changes in Thought study. Diagnostic evaluation of each case was performed by a board-certified neuropathologist in the NPTMT Core at the time of autopsy in accordance with the NIA-AA guidelines [1], which require assignment of an "ABC" score designating each subject as having "not", "low", "intermediate", or "high" AD neuropathologic change [2]. In order to test a range of $\mathrm{AD}$ pathologic change, we selected 9-10 cases from not/low, intermediate, and high categories that were matched by age, sex, postmortem interval, and year of death (see Table 1).

\section{Preparation of antibody-conjugated Luminex ${ }^{\mathrm{Tm}}$ beads}

Coupling Luminex MagPlex-COOH beads (Bio-Rad; Hercules, CA) to monoclonal antibodies was based on the procedures outlined in the X-MAP Cookbook [15]. All primary antibodies that contained amine-containing additives or preservatives were cleaned using Micro Bio-Spin 6 Tris chromatography columns (Bio-Rad; Hercules, CA) according to the manufacturer's instructions. Table 2 lists the antibodies that were conjugated to MagPlex beads to detect A $\beta 42$, pTau, GFAP, and IBA1.

Conjugation of antibodies to Luminex ${ }^{\mathrm{TM}}$ MagPlex-COOH beads was performed using the Bio-Rad bead making kit (Bio-Rad; Hercules, CA), and conjugated beads were quantified using a TC20 cell counter (Bio-Rad; Hercules, CA). For confirmation of antibody coupling to beads, serial dilutions of phycoerythrin (PE)-labeled anti-species $\mathrm{IgG}$ detection 
Table 1 Neuropathologic, clinical and demographic characteristics of subjects

\begin{tabular}{llllll}
\hline & Group & $\begin{array}{l}\text { Number of } \\
\text { subjects }\end{array}$ & $\begin{array}{l}\text { Age in years } \\
(\text { mean } \pm \text { SD })\end{array}$ & $\begin{array}{l}\text { PMI in hours } \\
(\text { mean } \pm \text { SD) }\end{array}$ & $\begin{array}{l}\text { Sex }(\% \\
\text { female })\end{array}$ \\
\hline Neuropathology & Not/low AD & 10 & $83.5 \pm 10.51$ & $5.03 \pm 1.59$ & 70 \\
& Intermediate AD & 10 & $89 \pm 5.21$ & $4.48 \pm 1.73$ & 60 \\
& High AD & 9 & $86.4 \pm 5.36$ & $5.26 \pm 1.53$ & 80 \\
Clinical & No dementia & 12 & $85.78 \pm 8.50$ & $4.90 \pm 1.59$ & 83.3 \\
& Dementia & 17 & $87.08 \pm 6.08$ & $4.96 \pm 1.60$ & 58.8 \\
Genetic & No APOE- $\varepsilon 4$ allele & 18 & $88.95 \pm 6.22$ & $4.80 \pm 1.63$ & 61.1 \\
& $\geq 1$ APOE- $\varepsilon 4$ allele & 11 & $81.73 \pm 7.67$ & $5.13 \pm 1.51$ & 81.8 \\
\hline
\end{tabular}

Table 2 Antibodies that were conjugated to magnetic beads to capture antigens

\begin{tabular}{lllll}
\hline Antigen & Product code & Manufacturer & Host & Clone \\
\hline A 342 & 700254 & Invitrogen & Rabbit & H31L21 \\
pTau & MN1020 & Invitrogen & Mouse & AT8 \\
GFAP & ab218309 & Abcam & Rabbit & EPR1034Y \\
IBA1 & ab221790 & Abcam & Rabbit & EPR16589
\end{tabular}

A 42 amyloid beta 42, pTau phospho-tau, GFAP glial fibrillary acidic protein, IBAI ionized calcium-binding adapter molecule 1

antibodies (Jackson ImmunoResearch; West Grove, PA) were used to detect primary bead-coupled antibodies from mouse or rabbit hosts (Table 2). The beads were then read using the Bio-Plex 200 Luminex instrument (Bio-Rad; Hercules, CA).

\section{Preparation of biotinylated antibodies}

The Luminex assay described in this manuscript uses a sandwich-assay format that requires two antibodies for each antigen. MagPlex bead-conjugated antibodies described in the previous section and in Table 2 served as antigen-capture antibodies and biotinylated antibodies (see Table 3 for list of detection antibodies) as detection antibodies. GFAP and IBA1 antibodies were obtained as biotin conjugates from their respective manufacturers (both Abcam; Cambridge, MA). Antibodies for detection of A $\beta 42$ (Biolegend; San Diego, CA), and Tau (Thermo Fisher Scientific; Waltham, MA) were biotinylated in our laboratory using a One-step Antibody Biotinylation kit (Miltenyi; Auburn, CA).

\section{Generation of recombinant $A \beta 42$ peptide}

Coding sequences for green-fluorescent protein in frame with 2 A peptide from porcine teschovirus-1 polyprotein and with a fusion construct encoding the first 243 amino acids of BRI followed by 42 amino acids encoding A 342 were cloned into an expression plasmid under the control of a human polypeptide elongation-factor $1 \alpha$ promoter and containing a woodchuck hepatitis-virus post-transcriptional regulatory element, and a human growth hormone
Table 3 Antibodies that were biotinylated to detect captured antigens

\begin{tabular}{lllll}
\hline Antigen & Product code & Manufacturer & Host & Clone \\
\hline A $\beta 42$ & 803001 & Biolegend & Mouse & 6E10 \\
Tau & MN1000 & Invitrogen & Mouse & HT7 \\
GFAP & LS-B2645-50 & LS BioSciences & Goat & NA (polyclonal) \\
IBA1 & MAB3402B & EMD Millipore & Mouse & GA5 \\
\hline
\end{tabular}

A 442 amyloid beta 42, GFAP glial fibrillary acidic protein, IBAI ionized calcium binding adapter molecule 1

polyadenylation signal. A similar strategy has been successfully used for expression of secreted $\mathrm{A} \beta$ peptides in cell lines and rats [21, 22]. We then transfected HEK cells with the A $\beta 42$ expression plasmid using polyethylenimine (PEI, Polysciences; Warrington, PA) transfection. Cells were incubated with the DNA/PEI mix for $12 \mathrm{~h}$, after which we removed the medium containing the DNA/PEI mix and replaced it with growth medium (DMEM, $10 \%$ fetal bovine serum, penicillin/streptomycin-all from Life Technologies; Carlsbad, CA). Seventy-two hours after the transfection we collected the cell supernatant, removed cell contaminants by centrifugation $(1200 \mathrm{rpm})$, sterile-filtered the supernatant, added sodium azide $(0.09 \%)$ for preservation, and stored aliquots at $-20{ }^{\circ} \mathrm{C}$.

\section{Protein extraction of formalin-fixed paraffin- embedded brain tissue}

Autopsy brain tissue was immersion fixated in $10 \%$ neutral buffered formalin for 2-3 weeks. Brain regions containing Brodmann area 9 of middle frontal gyrus (MFG), hippocampus at the level of the corpus geniculatum laterale, and neostriatum at the level of the anterior commissure, were then sampled, embedded in paraffin, microtome sliced in $15-\mu \mathrm{m}$ sections, and placed on charged microscope slides [13]. Three adjacent $15-\mu \mathrm{m}$ sections per case were deparaffinized by immersion for $3 \mathrm{~min}$ in each of the following solutions: $3 \times$ in $100 \%$ Xylene, $100 \%$ isopropanol, $96 \%$ isopropanol, $70 \%$ isopropanol, and 50\% isopropanol. Sections were then washed in PBS containing $0.05 \%$ Tween $2 \mathrm{x}$ for 4 min [14]. For antigen retrieval, sections were boiled 
for $20 \mathrm{~min}$ in $0.1 \mathrm{M}$ citrate buffer at a $\mathrm{pH}$ of 6.1. Slides were then immersed in PBS containing $0.05 \%$ Tween. Tissue from three sections was then scraped off the slides with a razor blade and placed into a 2-ml tube.

For extraction of $A \beta 42$ and pTau, $150 \mu \mathrm{l}$ of chilled RIPA buffer ( $1 \%$ NP-40, $0.5 \%$ sodium deoxycholate, $150 \mathrm{mM}$ sodium chloride, $50 \mathrm{mM}$ Tris hydrochloride, $0.5 \mathrm{mM}$ magnesium sulfate-all from Sigma-Aldrich; St. Louis, MO) with Complete Mini protease inhibitor (Sigma-Aldrich; St. Louis, MO) was immediately added to the tube. Samples were then sonicated on ice (10 pulses, $3 \times$ ), and centrifuged for $45 \mathrm{~min}$ at $21,000 \times g$ and $4{ }^{\circ} \mathrm{C}$. Supernatants containing RIPA-soluble proteins were pipetted off into new $2 \mathrm{ml}$ tubes. The remaining pellet was washed with an additional $50 \mu$ RIPA buffer with Complete Mini, and centrifuged a second time. The RIPAbuffer containing supernatant was then combined with the first RIPA-buffer containing supernatant. The combined RIPA-buffer supernatant contains soluble proteins and pTau, but not $A \beta 42$. For $A \beta 42$ extraction, $150 \mu$ lo chilled $5 \mathrm{M}$ guanidine-hydrochloride $(\mathrm{Gu}-\mathrm{HCl})$ buffer containing Complete Mini was then added to the remaining pellet in tube, vortexed, then sonicated on ice for 10 pulses. Centrifugation at $13,000 \times g$ for $30 \mathrm{~min}$ at $4{ }^{\circ} \mathrm{C}$ produced a $\mathrm{Gu}-\mathrm{HCl}$ soluble supernatant containing the $\mathrm{A} \beta 42$ fraction and other RIPA insoluble proteins. All extracts were stored at $-80^{\circ} \mathrm{C}$.

IBAI and GFAP were extracted from deparaffinized sections using an FFPE extraction kit (Qiagen; Germantown, MD) by following the 2D-PAGE/MS protocol of the kit instructions. The resulting protein extract was dissolved in $100 \mu \mathrm{l} \mathrm{RIPA}$ with Complete Mini. All extracts were stored at $-80^{\circ} \mathrm{C}$.

Total protein content was determined in all samples using a BCA kit (Pierce; Rockford, IL) together with colorimetric detection (absorbance at $562 \mathrm{~nm}$ ) in a plate reader.

\section{Luminex assay using customized antibodies and MagPlex beads}

Protein samples and standards were always diluted in PBS containing $1 \%$ bovine serum albumin (BSA, SigmaAldrich; St. Louis, MO) and conjugated MagPlex beads were always diluted in PBS with $1 \%$ BSA and Complete Mini. Prior to adding conjugated MagPlex beads to samples, beads were thoroughly resuspended by vortexing and sonication for $20 \mathrm{~s}$ each. Diluted samples and standards were then loaded onto a black flat-bottom 96-well plate (Greiner; Monroe, NC), mixed with resuspended MagPlex beads at a concentration of 2500 beads per well, and incubated overnight. This and all following incubation steps were performed shaking in the dark and at room temperature. On the next day, beads were washed twice and then incubated for 3-4 h with $100 \mathrm{ng}$ of biotinylated antibody (in PBS with $1 \%$ BSA) per well. After that, beads were washed twice and then incubated for $1 \mathrm{~h}$ with $1 \mu \mathrm{g}$ of PE per well (Life Technologies; Carlsbad, CA). After that beads were washed twice, resuspended in PBS, incubated for $5 \mathrm{~min}$, and then read using the Bio-Plex 200 Luminex instrument.

For analysis of $A \beta 42$, we first used different amounts of $\mathrm{Gu}-\mathrm{HCl}$ soluble protein extracts to determine the optimal amount of sample (based on total protein content) per well, and then used that amount for the analysis of all experimental samples.

For analysis of pTau, we first used different amounts of RIPA-soluble protein extracts to determine the optimal amount of sample (based on total protein content) per well, and then used that amount for the analysis of all experimental samples.

For analysis of IBAI and GFAP, we first used different amounts of RIPA-soluble protein extracts (Qiagen-kit) to determine the optimal amount of sample (based on total protein content) per well, and then used that amount for the analysis of all samples.

\section{Luminex assay using the ALZ kit}

Recombinant $\mathrm{A} \beta 42$ was also measured using the INNOBIA AlzBio3 kit (Fujirebio through INNOGENETICS; Gent, Belgium) according to the manufacturer's instructions.

\section{Commercial standards used for GFAP and IBA1 assays}

For GFAP and IBA1 Luminex assays we identified suitable standards that we used for validation purposes and which will allow quantification of GFAP and IBA1 in future experiments. For GFAP we used porcine full-length protein purified from spinal cord (Millipore; Burlington, MA) and for IBA1 we used full-length recombinant human IBA1 (Abcam; Cambridge, MA). We prepared the following concentrations for GFAP: 320,000, 160,000, 80,000, $40,000,20,000,10,000,5000,2500,1250$, and 625 . For IBA1 we prepared the following concentrations: 40,000 , $20,000,10,000,5000,2500,1250,625$, and $312.5 \mathrm{pg} / \mathrm{ml}$.

\section{Measuring gray-matter area using IHC and image analysis}

Because most of the proteins measured in this study are known to be differentially distributed between gray and white matter, we needed to control for variability in the gray/white matter composition between samples. To accomplish that, $5 \mu \mathrm{m}$ sections immediately anterior to sections utilized for protein extraction were treated with Epitope Retrieval Solution 2 (Leica Biosystems, Buffalo Grove, IL) for $20 \mathrm{~min}$, immunostained for synaptophysin 
Fig. 1 Validation of recombinant $\mathrm{A} \beta 42$ standards. a Quantification of recombinant A $\beta 42$ using commercial Innogenetics-kit standards and the Innogenetics kit. b

Comparison of serial dilutions of Innogenetics-kit and recombinant $A \beta 42$ standards measured with the Innogenetics kit. c Generation of a standard curve with recombinant A $\beta 42$ standards measured with our newly developed Luminex assay. d Test-retest of serially diluted recombinant

A $\beta 42$ standards measured with our newly developed Luminex assay. All data are presented as mean \pm SEM
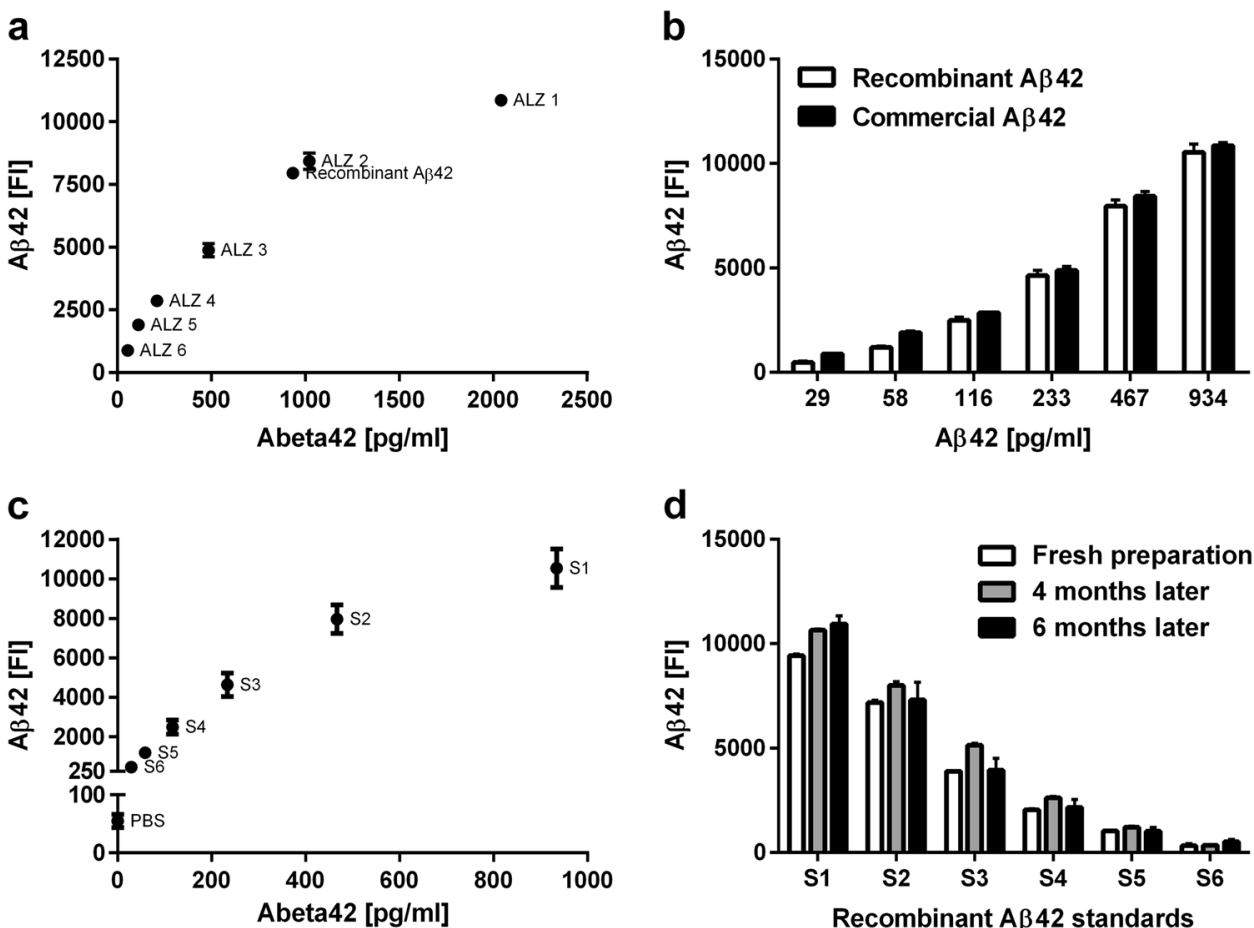

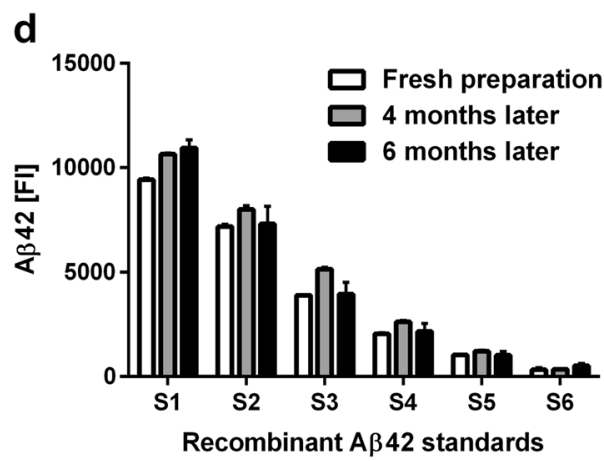

with rabbit monoclonal MRQ-40 antibody (1:100, Cell Marque, Rocklin, CA), and counterstained with hematoxylin. The sections were then scanned at $\times 40$ magnification with an Aperio AT2 digital slide scanner (Leica Biosystems, Buffalo Grove, IL) and subjected to histomorphologic analysis using Aperio GENIE Histology Pattern Recognition software (Leica Biosystems, Buffalo Grove, IL) to differentiate between synaptophysin immunoreactive regions and calculate the gray matter area of each section. We used this procedure to control for potential differences of gray matter area in this analysis cohort.

\section{Statistical analysis}

All experimental and basic patient data are shown as mean \pm standard error of the mean (SEM). Data were analyzed using one-way or two-way analysis of variance (ANOVA), followed by Tukey's post-test, or a two-tailed $t$-test. Correlations were evaluated by calculating Pearson's correlation coefficient, and linearity was assessed by linear regression. Alpha was always set at 0.05 .

\section{Results}

\section{Generation of recombinant standards for quantification of $\mathrm{A} \beta 42$}

To assess whether we could generate recombinant $\mathrm{A} \beta 42$ standards for the quantification of $\mathrm{A} \beta 42$, we transfected HEK cells with pGFP-BRI-A $\beta 42$ and used the commercial Innogenetics Luminex kit to detect and quantify $\mathrm{A} \beta 42$ in the supernatant of transfected cells (72-h post transfection). We confirmed the presence of $A \beta 42$ in the supernatant of transfected cells and measured an $\mathrm{A} \beta 42$ concentration of $934 \mathrm{pg} / \mathrm{ml}$ in the supernatant (Fig. 1a). Next, we tested serial dilutions of recombinant $A \beta 42$ and A $\beta 42$ standards from the Innogenetics kit (each at 29, 58, $113,233,467$, and $934 \mathrm{pg} / \mathrm{ml}$ ) using the Innogenetics kit for detection and quantification (Fig. 1b). Analysis of fluorescent intensities of diluted recombinant $\mathrm{A} \beta 42$ and A $\beta 42$ standards from the Innogenetics kit by two-way ANOVA revealed a significant effect of A $\beta 42$ concentration $\left(F_{5,36}=321.7, \quad p<0.01\right)$, with significant differences between different $\mathrm{A} \beta 42$ dilutions for both recombinant $\mathrm{A} \beta 42$ and $\mathrm{A} \beta 42$ standards $(p<0.05)$, but with no significant differences between recombinant and commercial $\mathrm{A} \beta 42$ standards at equal dilutions $(p>0.05)$. We then tested our recombinant $\mathrm{A} \beta 42$ standards with the newly developed Luminex A $\beta 42$ assay (capture with H31L21 clone, detection with $6 \mathrm{E} 10$ clone). Using the same concentrations of recombinant $A \beta 42$ that we analyzed with the Innogenetics Luminex kit, we were able to generate a standard curve (Fig. 1c) similar to the one generated by commercial A $\beta 42$ standards. We designated our recombinant standards as S1 (934 pg/ml), S2 (467 pg/ml), S3 (233 pg/ml), S4 (113 $\mathrm{pg} / \mathrm{ml})$, S5 $(58 \mathrm{pg} / \mathrm{ml})$, and S6 $(29 \mathrm{pg} / \mathrm{ml})$. We further determined whether our recombinant $\mathrm{A} \beta 42$ standards could be stored and used over an extended period of time (Fig 1d). Two-way ANOVA of fluorescent intensities of 

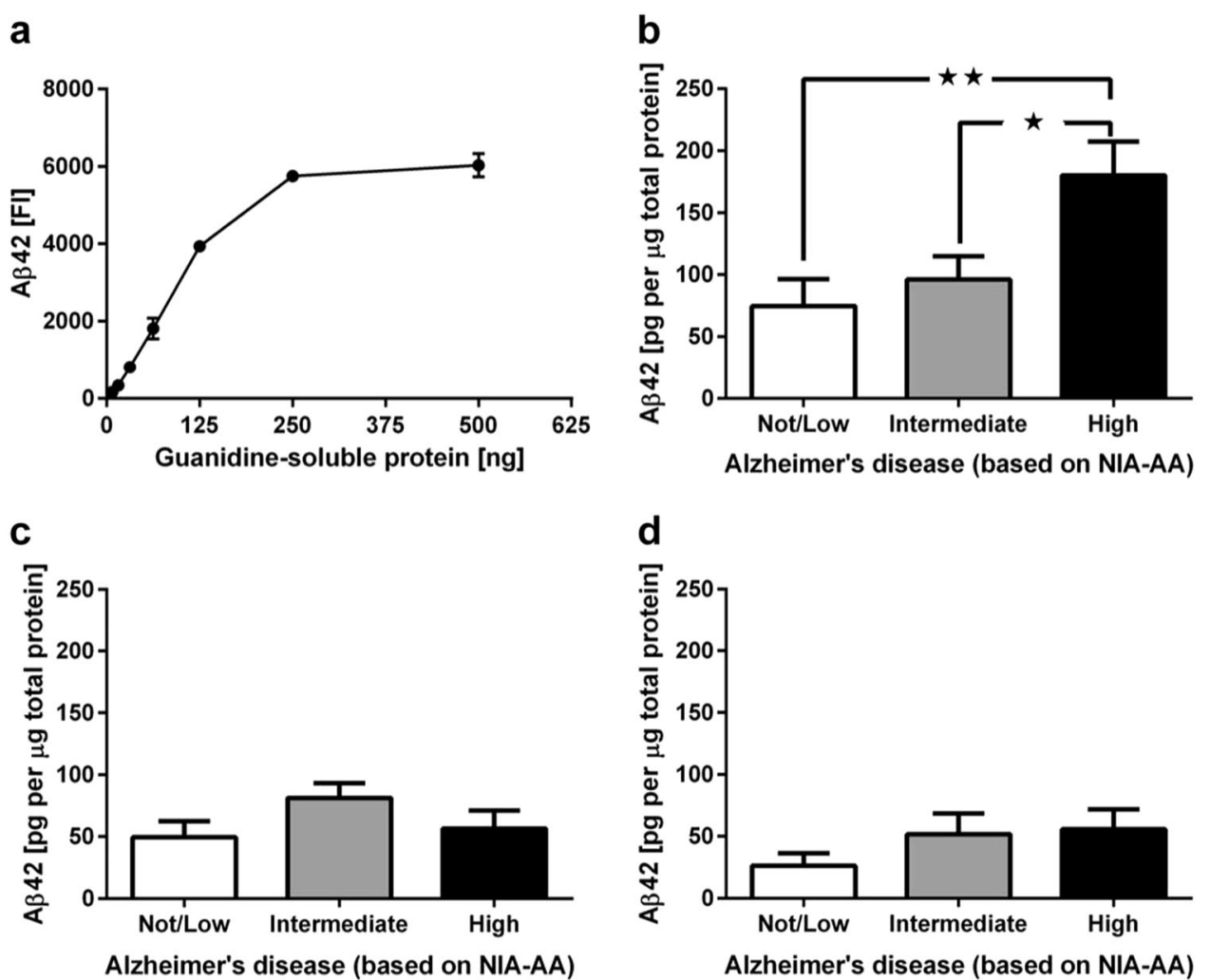

Fig. 2 Determination of optimal FFPE sample amounts for Luminex analysis of $\mathrm{A} \beta 42$, and quantification of $\mathrm{A} \beta 42$ in FFPE tissue from cases with not/low, intermediate, and high NIA-AA AD severity. a Fluorescent-intensity signals for $\mathrm{A} \beta 42$ as function of increasing amounts of guanidine-soluble extracts from middle frontal gyrus. b A $\beta 42$ levels in MFG samples from cases with varying NIA-AA AD severity (not/low: $n=10$, intermediate: $n=10$, high: $n=9$ ). c A $\beta 42$

levels in hippocampal samples from cases with varying NIA-AA AD severity (not/low: $n=10$, intermediate: $n=10$, high: $n=9$ ). d A $\beta 42$ levels in neostriatal samples from cases with varying NIA-AA AD severity (not/low: $n=10$, intermediate: $n=10$, high: $n=9$ ). A $\beta 42$ levels in panels (b-d) are normalized to the amount of guanidinesoluble protein $(\mathrm{pg} / \mu \mathrm{g})$. All data are presented as mean \pm SEM. $\star p<$ 0.05 and $\star \star p<0.01$

S1-S6 standards measured immediately after, 2 and 6 months after preparation of recombinant $A \beta 42$ revealed significant main effects of standard concentration $\left(F_{5,30}=\right.$ $197, p<0.01)$, but no significant effects of time passed between preparation of standards and measurement of fluorescent intensities $(p>0.05)$.

\section{Detection of $A \beta 42$ in human FFPE tissue}

To determine whether we could use the same antibodies that are routinely used in neuropathology IHC assessments for Luminex-based detection of $\mathrm{A} \beta 42$, we extracted $\mathrm{Gu}-\mathrm{HCl}$ soluble fractions from the MFG from 3 to 5 additional AD cases that were not part of the experimental cohort, and tested increasing amounts of each fraction for the presence of $\mathrm{A} \beta 42$. Increasing amounts of $\mathrm{Gu}-\mathrm{HCl}$ soluble protein extracts resulted in increasing fluorescent signal intensities in the A 342 assay (capture with H31L21 clone, detection with 6E10 clone), and these signal intensities were approximately linear for protein amounts between 15,625 and $250 \mathrm{ng}$, approximately spanning a 35-fold dynamic

range (Fig. 2a). Based on the observed input curve, we determined that $\sim 150 \mathrm{ng}$ of $\mathrm{Gu}-\mathrm{HCl}$ soluble proteins allows for a strong $A \beta 42$ signal that is well within the linear range of the assay.

\section{Quantification of $A \beta 42$ in FFPE tissue from AD patients and controls}

Next we measured $\mathrm{A} \beta 42$ levels in $\mathrm{Gu}-\mathrm{HCl}$ extracts from FFPE MFG, hippocampal, and neostriatal tissue from patients with not/low, intermediate, and high levels of $\mathrm{AD}$ pathology (assessed per NIA-AA criteria), and then compared $A \beta 42$ levels between samples for each brain region. Comparisons of $A \beta 42$ levels in the MFG (Fig. 2b) by ANOVA revealed significant effects of NIA-AA AD severity $\left(F_{2,26}=5.67, p<0.01\right)$. Post hoc comparison with Tukey's multiple comparison test confirmed significantly increased $\mathrm{A} \beta 42$ levels in samples with high $\mathrm{AD}$ severity when compared to samples with not/low $\mathrm{AD}(p<0.01)$ and to samples with intermediate $\mathrm{AD}$ severity $(p<0.05)$. Comparison of $\mathrm{A} \beta 42$ levels in the hippocampus (Fig. 2c) by 

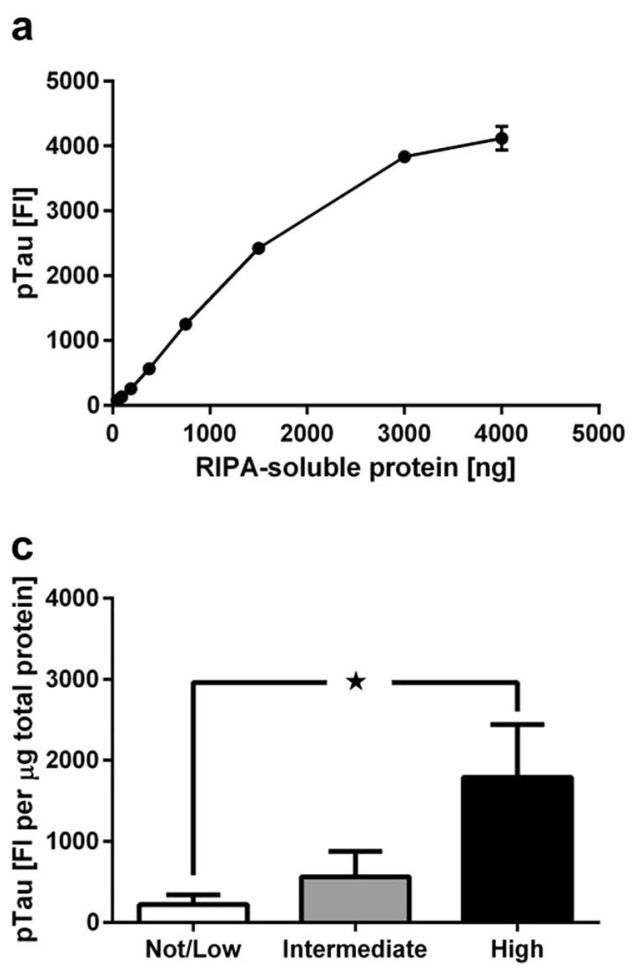

Alzheimer's disease (based on NIA-AA)

Fig. 3 Determination of optimal FFPE sample amounts for Luminex analysis of pTau, and quantification of pTau in FFPE tissue from cases with not/low, intermediate, and high NIA-AA AD severity. a Fluorescent-intensity signals for pTau as function of increasing amounts of RIPA-soluble extracts from middle frontal gyrus. b pTau levels in MFG samples from cases with varying NIA-AA AD severity (not/low: $n=10$, intermediate: $n=10$, high: $n=9$ ). c pTau levels in

ANOVA did not reveal significant effects of NIA-AA AD severity $(p>0.05)$. Although we saw visible trends for increased $A \beta 42$ levels in the neostriatum (Fig. 2d) of samples with intermediate and high NIA-AA AD severity, ANOVA of A $\beta 42$ levels in the neostriatum did not reveal statistically significant effects of NIA-AA AD severity $(p>$ $0.05)$.

\section{Detection of pTau in human FFPE tissue}

To determine whether we could use the same antibodies that are routinely used in neuropathology for Luminex-based detection pTau, we extracted RIPA soluble fractions from the MFG from 3 to 5 additional AD cases that were not part of the 30 cases/controls cohort, and tested increasing amounts of each fraction for the presence of pTau. Increasing amounts of RIPA-soluble protein extracts resulted in increasing fluorescent signal intensities in our pTau assay (capture with AT8 clone, detection with HT7 clone), and these signal intensities were approximately linear for protein amounts between 187.5 and $3000 \mathrm{ng}$, approximately spanning a 29-fold dynamic range (Fig. 3a). Based on the
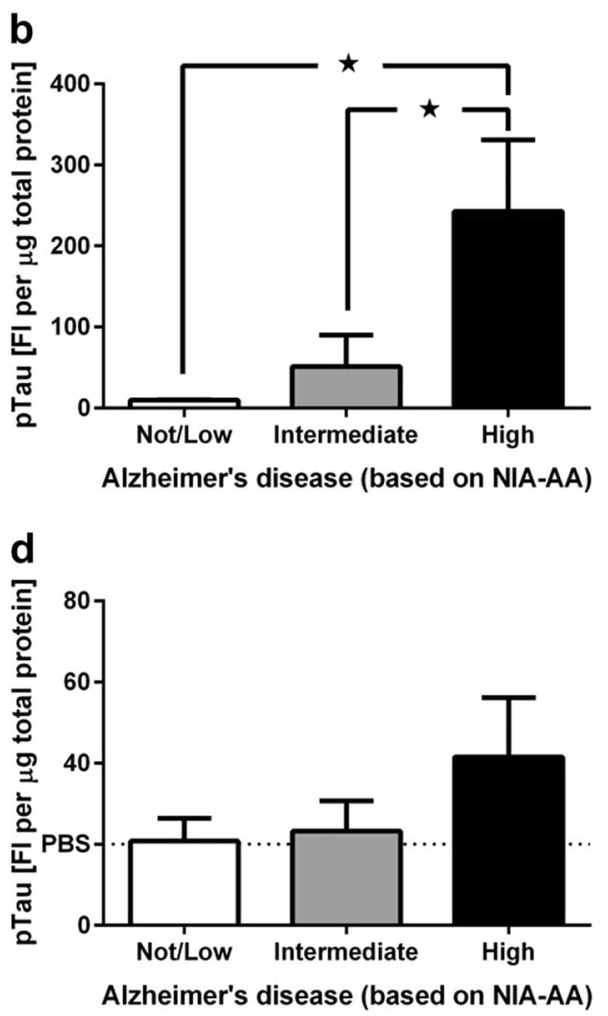

hippocampal samples from cases with varying NIA-AA AD severity (not/low: $n=10$, intermediate: $n=10$, high: $n=9$ ). d pTau levels in neostriatal samples from cases with varying NIA-AA AD severity (not/low: $n=10$, intermediate: $n=10$, high: $n=9$ ). pTau levels in panels (b-d) are normalized to the amount of RIPA-soluble protein (fluorescent intensity per $\mu \mathrm{g}$ ). All data are presented as mean \pm SEM. $\star p<0.05$ and $\star \star p<0.01$

observed input curve, we determined that $2000 \mathrm{ng}$ of RIPA soluble proteins allows for a strong pTau signal that is well within the linear range of the assay.

\section{Quantification of pTau in FFPE tissue from AD patients and controls}

We measured levels of pTau in RIPA extracts from the same FFPE MFG, hippocampal, and neostriatal tissue that we used for measuring $A \beta 42$, and then compared pTau levels between samples that were grouped based on NIA-AA AD severity. ANOVA of MFG pTau levels (Fig. 3b) revealed significant effects of NIA-AA AD severity $\left(F_{2,26}=4.96, p<0.01\right)$, and post hoc pair-wise comparisons with Tukey's multiple comparison test further showed that pTau levels in the group with high NIA-AA $\mathrm{AD}$ severity were significantly higher than in the groups with not/low and intermediate NIA-AA AD severity (each $p<0.05$ ). Similarly, comparison of pTau levels in the hippocampus (Fig. 3c) by ANOVA revealed significant effects of NIA-AA AD severity $\left(F_{2,25}=3.82, p<0.05\right)$. Post hoc pair-wise comparisons with Tukey's multiple comparison 
Fig. 4 Scatter plots of Luminex fluorescent signal intensities for A $\beta 42$ and pTau in MFG, hippocampus, and neostriatum. We performed correlation analyses of $\mathrm{A} \beta 42$ and pTau signals across all analyzed brain regions and confirmed the following statistically significant positive correlations. a A $\beta 42$ levels in MFG vs. $A \beta 42$ levels in hippocampus. b A $\beta 42$ levels in MFG vs. A $\beta 42$ levels in neostriatum. c A $\beta 42$ levels in hippocampus vs. $A \beta 42$ levels in neostriatum. d A $\beta 42$ levels in neostriatum vs. pTau levels in MFG. e pTau levels in MFG vs. pTau levels in hippocampus. Statistically significant $R^{2}$ values are shown in each scatter plot a

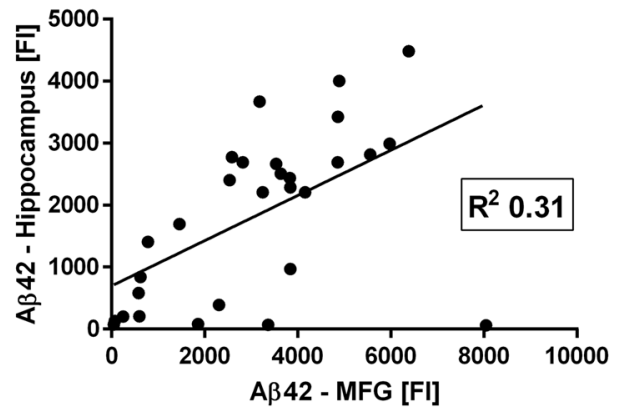

b

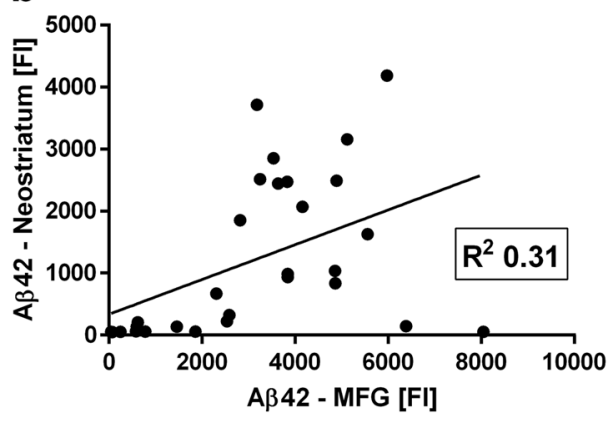

C

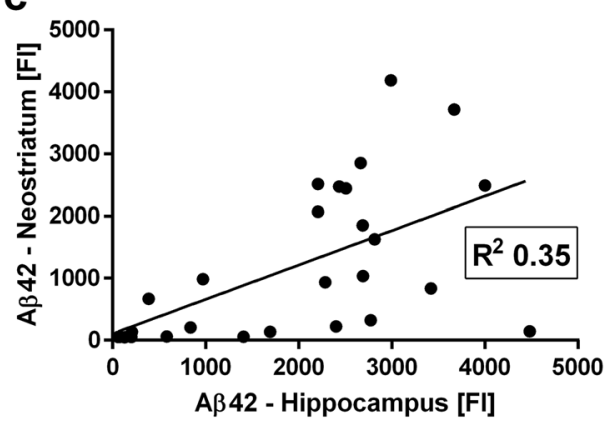

d

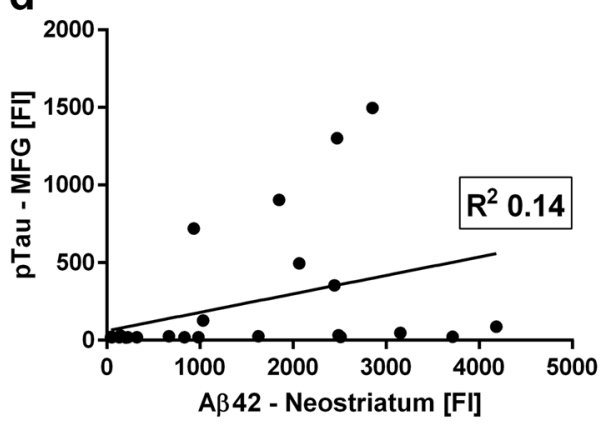

e

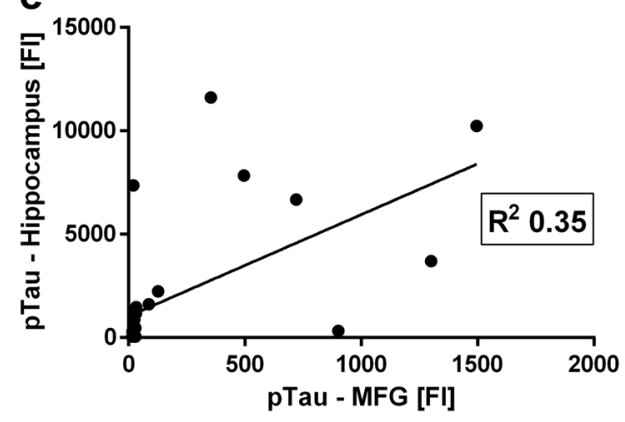

test further show that pTau levels in the group with high NIA-AA AD severity were significantly higher than in the group with not/low NIA-AA AD severity $(p<0.05)$. In contrast, comparison of pTau levels in the neostriatum (Fig. 3d) by ANOVA did not reveal any significant effects of NIA-AA AD severity (each $p>0.05$ ). We noticed that pTau signal in most samples from the neostriatum was at or close to the level of PBS reagent blanks.

\section{Correlation analysis of A 342 and pTau levels in FFPE sections from $A D$ patients and controls}

We performed correlation analyses of $\mathrm{A} \beta 42$ and pTau levels in all analyzed brain regions. A $\beta 42$ levels in the MFG were correlated positively with $A \beta 42$ levels in hippocampus (Fig. 4a) and neostriatum (Fig. 4b), and A $\beta 42$ levels in the hippocampus were correlated positively with $A \beta 42$ levels in the neostriatum (Fig. 4c). A $\beta 42$ levels in the neostriatum were correlated positively with pTau levels in the MFG
(Fig. 4d). pTau levels in the MFG were correlated positively with pTau levels in hippocampus (Fig. 4f). Although pTau levels in the neostriatum correlated significantly with A $\beta 42$ and pTau levels in some other brain regions, these correlations cannot be interpreted because pTau levels in the neostriatum were essentially as low as PBS blanks. All correlations, including not statistically significant correlations and correlations with pTau in the neostriatum are listed in Table 4.

\section{Gray-matter area measurements in all FFPE sections from AD patients and controls}

In order to potentially correct for variability in white matter content in tissue sections (with the vast majority of $\mathrm{A} \beta 42$ and pTau expected in cortex), we measured the area of gray matter in sections that were immediately adjacent to all FFPE sections that we used for protein extractions for the Luminex assays (Supplemental Fig. 1). Comparisons of 
Table 4 Correlations $\left(R^{2}\right)$ between $\mathrm{A} \beta 42$ and pTau levels in MFG, hippocampus, and neostriatum in all analyzed samples. Statistically significant correlation coefficients are marked: $* p<0.05$ and $* * p<$ 0.01

\begin{tabular}{|c|c|c|c|c|c|c|c|}
\hline & & $\mathrm{A} \beta 42$ & & & pTau & & \\
\hline & & $\begin{array}{l}\text { MF- } \\
\text { G }\end{array}$ & HPC & NEO & MFG & HPC & NEO \\
\hline $\mathrm{A} \beta 42$ & MFG & - & $0.31 * *$ & $0.21 *$ & 0.01 & 0.01 & 0.09 \\
\hline & HPC & - & - & $0.35 * *$ & 0.06 & 0.02 & 0.01 \\
\hline & NEO & - & - & - & $0.14 *$ & 0.11 & $0.30 * *$ \\
\hline pTau & MFG & & & & - & $0.35^{* *}$ & $0.23 * *$ \\
\hline & HPC & & & & & - & $0.23 * *$ \\
\hline & NEO & & & & & & - \\
\hline
\end{tabular}

A 442 amyloid beta 42, pTau phospho-tau, $M F G$ middle frontal gyrus, $H P C$ hippocampus, NEO neostriatum

gray matter areas in MFG, hippocampus, and striatum by ANOVA did not reveal any significant effects of NIA-AA AD severity $\left(\mathrm{F}_{2,79}=2.7, \mathrm{p}>0.05\right)$.

\section{Association of quantitative measurements of $A \beta 42$ and pTau with genetic risk for AD}

$A P O E-\varepsilon 4$ is the strongest genetic risk factor for sporadic $\mathrm{AD}$ with increased risk with one or two copies of the $A P O E-\varepsilon 4$ allele [23]. Our validation cohort included 19 cases without the $A P O E-\varepsilon 4$ and 11 cases with at least one $A P O E-\varepsilon 4$ allele. Comparisons of $A \beta 42$ levels in carriers and non-carriers of the $A P O E-\varepsilon 4$ allele across all sampled brain regions (Fig. 5a) by ANOVA revealed a significant effect of APOE genotype on A $\beta 42$ levels $\left(F_{1,82}=6.13, p<0.05\right)$. However, post hoc pair-wise comparisons failed to show significant $(p>0.05)$ mean differences between specific groups. Comparisons of pTau levels in carriers and noncarriers of the APOE- 44 allele across all sampled brain regions (Fig. 5b) by ANOVA did not reveal a significant effect of $A P O E$ genotype on pTau levels $\left(F_{1,82}=0.76, p>\right.$ $0.05)$.

\section{Association of quantitative measurements of $A \beta 42$ and pTau with ante-mortem cognitive status}

Quantitative measures of $\mathrm{AD}$ pathology can be used to probe for associations between $\mathrm{A} \beta 42$ and pTau and cognitive status (no dementia vs. dementia). We compared $A \beta 42$ and pTau levels in cases with and without dementia, and found that cases with dementia had significantly higher A $\beta 42$ and pTau levels in MFG and significantly higher A $\beta 42$ levels in the neostriatum than non-demented cases ( $p$ $<0.05$, Supplemental Fig. 2). Hippocampal levels of A $\beta 42$ and pTau were not significantly higher in demented vs. nondemented cases ( $p>0.05$, Supplemental Fig. 2); although
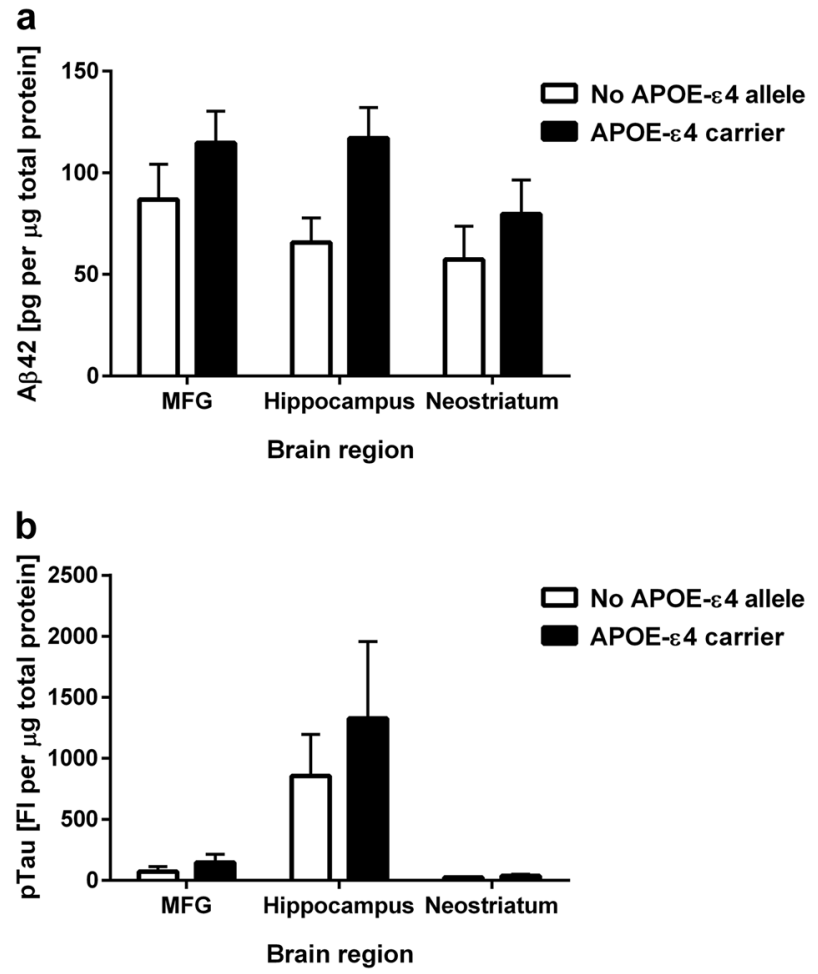

Fig. 5 Association of $A \beta 42$ and pTau levels with genetic risk for AD. a A $\beta 42$ and b pTau levels in MFG, hippocampus, and neostriatum of samples from cases with no $A P O E-\varepsilon 4$ allele $(n=18)$ and with at least one $A P O E$ - $\varepsilon 4$ allele $(n=11)$. All data are presented as mean \pm SEM

there was a trend for increased hippocampal pTau in demented subjects. pTau levels in the neostriatum were too low to be included in this analysis.

\section{Detection of GFAP and IBA1 in human FFPE tissue}

To determine whether we could use Luminex-based technology to probe for pathologic pathways in FFPE MFG, we first validated GFAP and IBA1 Luminex reagents with commercially available GFAP (Fig. 6a) and IBA1 (Fig. 6b) peptides. Increasing concentrations of peptides resulted in increasing fluorescent signal intensities in both GFAP (capture with EPR1034Y clone, detection with polyclonal antibody) and IBA1 (capture with EPR16589 clone, detection with GA5 clone) assays, and these signal intensities were approximately linear for peptide concentrations between 5000 and $160,000 \mathrm{pg} / \mathrm{ml}$ for GFAP and between 300 and $40,000 \mathrm{pg} / \mathrm{ml}$ for IBA1. Next we extracted RIPA and $\mathrm{Gu}-\mathrm{HCl}$ soluble fractions from MFG from 3 to 5 additional $\mathrm{AD}$ cases that were not part of our 30 cases/ controls cohort, and tested increasing amounts of each fraction for the presence of GFAP and IBA1. No signals could be detected for GFAP and IBA1 in these fractions (data not shown). However, when we used MFG protein lysates extracted with a commercial FFPE extraction kit 
Fig. 6 Luminex analysis of GFAP and IBA1. a Generation of a standard curve with commercial GFAP standards. b Generation of a standard curve with commercial

IBA1 standards. c GFAP fluorescent-intensity signals for increasing amounts of FFPE middle frontal gyrus extracts. d IBA1 fluorescent-intensity signals for increasing amounts of FFPE middle frontal gyrus extracts. All extractions were made with the commercial Qiagen FFPE kit, and extracted proteins were resuspended in RIPA buffer. All fluorescent intensities were measured with our newly developed Luminex assay
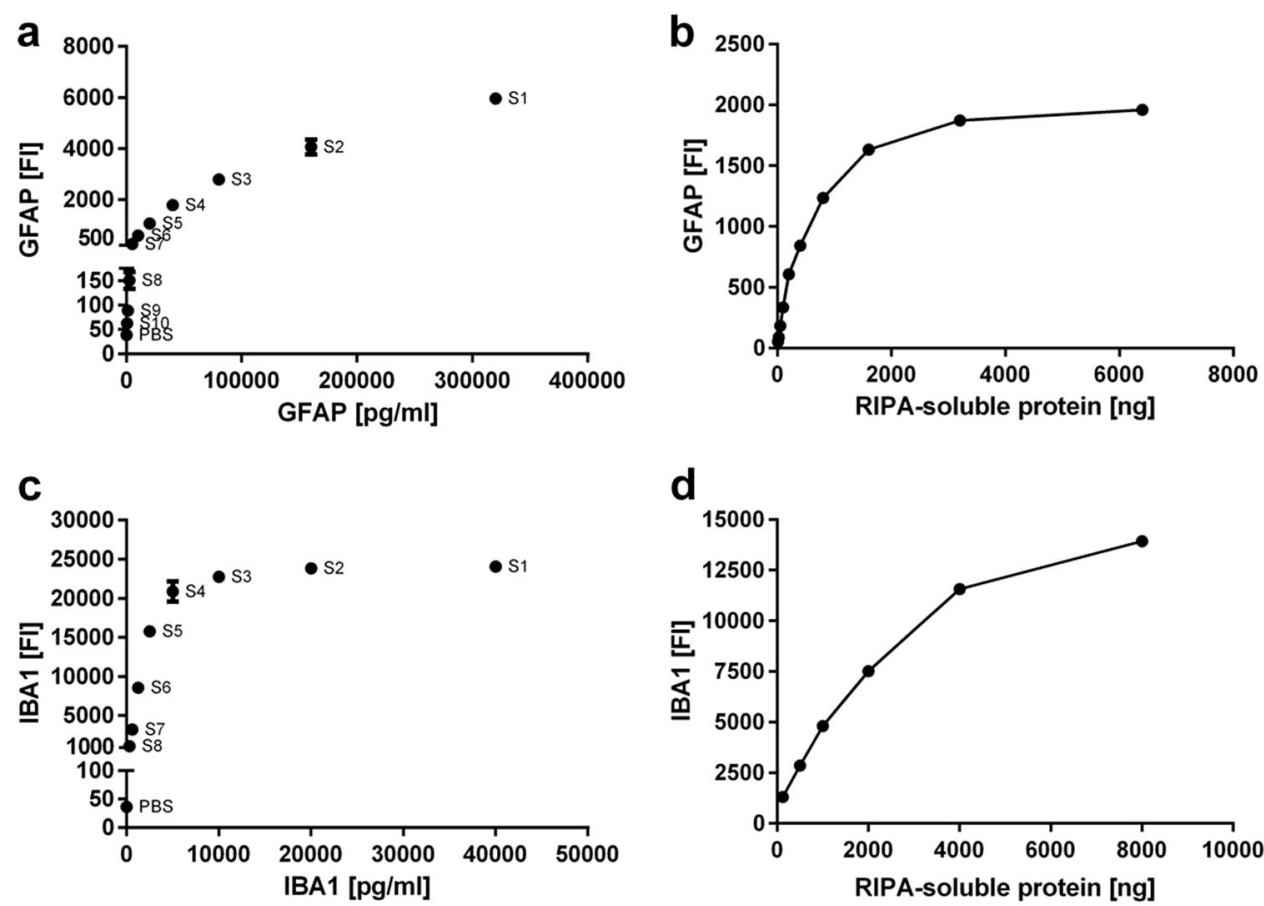

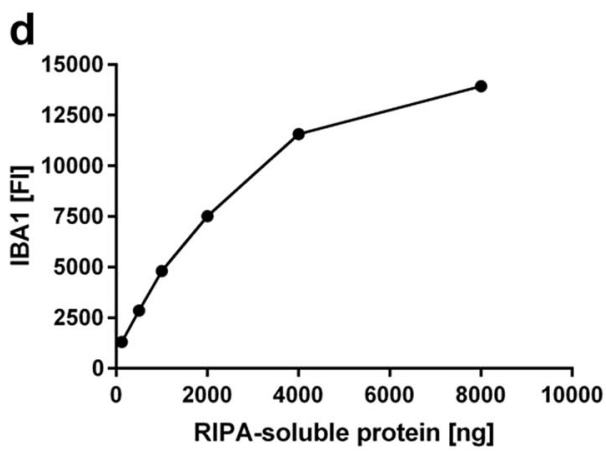

(Qiagen), we were able to detect GFAP and IBA1. Increasing amounts of Qiagen protein extracts resulted in increasing fluorescent signal intensities in the GFAP assay, and these signal intensities were approximately linear for protein amounts between 100 and $3200 \mathrm{ng}$ (Fig. 6c). Similarly, increasing amounts of Qiagen protein extracts resulted in increasing fluorescent signal intensities in the IBA1 assay, and these signal intensities were approximately linear for protein amounts between 125 and $4000 \mathrm{ng}$ (Fig. 6d).

\section{Discussion}

Here, we describe the development of a Luminex-based method to generate highly quantitative, molecularly specific measurements of pathologic peptides and reactive and inflammatory proteins in FFPE brain tissue.

A major obstacle to the analysis of FFPE protein lysates is the formation of methylene bridges between side chains of proteins, cross-linking, and preserving the peptides of cellular proteins. To establish an FFPE-compatible Luminex assay, we first developed procedures to solubilize $\mathrm{A} \beta 42$ and pTau in protein extracts from FFPE tissue. We based these procedures on immunohistology protocols that are routinely used for antigen retrieval and subsequent immunostaining for $A \beta 42$ and pTau [13]. We found that deparaffinization followed by citric-acid boil, homogenization in RIPA buffer and sequential extraction with RIPA buffer (solubilizes pTau) and then $\mathrm{Gu}-\mathrm{HCl}$ (solubilizes $\mathrm{A} \beta 42$ ) produced protein extracts that could be quantified using standard protein quantification kits and that resulted in robust Luminex detection of $A \beta 42$ and pTau. As a result, this method allows to standardize downstream analyses through normalization of measured signal intensities to total amounts of protein per analyzed fraction (e.g. RIPA-soluble or $\mathrm{Gu}-\mathrm{HCl}$ soluble fractions). By varying the amount of total protein analyzed, we obtained signal intensities that were almost perfectly linear over a 35 -fold dynamic range for $\mathrm{A} \beta 42$ and almost perfectly linear over a 29-fold dynamic range for pTau, which are in good agreement with several published studies [13, 14, 24-27]. To align with clinical and research neuropathologic assessments in $\mathrm{AD}$, we chose $\mathrm{A} \beta 42$ and pTau antibodies that are currently recommended for standard of care.

We generated a cell-culture-based source of A 342 standards that performed, as well as commercial A $\beta 42$ standards when used with a widely used commercial A 342 Luminex assay (INNO-BIA AlzBio3 kit), which then allowed the generation of standard curves for the quantification of $A \beta 42$ with our own Luminex assay. The relative ease of preparation and the verified shelf life of at least 6 months makes our cell-culture-based A $\beta 42$ standard a useful and cost-effective alternative to commercially available A $\beta 42$ standards.

We then generated protein preparations from FFPE brain samples obtained from 30 autopsies of research subjects with $\mathrm{AD}$ neuropathologic change ranging from not/low to intermediate and high $\mathrm{AD}$ pathologic change (NIA-AA criteria). We used samples collected according to NIA-AA recommendations for $\mathrm{AD}$ and included MFG, hippocampus, 
and neostriatum in our analysis [2]. Consistent with the NIA-AA-based neuropathologic diagnosis of AD, the Luminex assay detected low levels of A $\beta 42$ and pTau in MFG samples with low levels of AD neuropathologic change, and higher levels of $\mathrm{A} \beta 42$ and pTau in MFG samples with high levels of $\mathrm{AD}$ neuropathologic change. Also in strong agreement with the NIA-AA-based neuropathologic diagnosis of $\mathrm{AD}$, Luminex measurements confirmed significantly higher hippocampal pTau levels in cases with high NIA-AA AD neuropathologic change. While the Luminex assay detected upwards trending levels of $A \beta 42$ in the neostriatum of cases with high NIA-AA AD neuropathologic change, we could not detect significant levels of neostriatal pTau. Taken together, our quantitative Luminex data are in solid agreement with A $\beta 42$ and pTau neuropathologic changes typical of NIA-AA AD severity. Although we detected strong differences between the not/ low and high NIA-AA AD neuropathologic change groups, we only observed trends for increased $A \beta 42$ and pTau levels in cases with intermediate NIA-AA AD neuropathologic change. Increasing sample sizes in future studies will most likely resolve this issue.

We observed, that the levels of $A \beta 42$ in MFG were roughly twice as high as in either the hippocampus or the neostriatum, and pTau levels in the hippocampus were almost 10 times higher than in the MFG. Hence, this method not only allows detection of inter-individual disease-associated levels of A $\beta 42$ and pTau across the same brain regions, but also intra-individual differences between various brain regions and absolute quantitation of target protein/peptide when standards are available.

We performed a simple and preliminary analysis of the association between quantitative assessments of $\mathrm{A} \beta 42$ and pTau with ante-mortem clinical diagnosis of dementia, and found elevated levels of A $\beta 42$ and pTau in samples from demented individuals. We are aware that our validation cohort has not been specifically selected for such an association study, but are encouraged by this current finding to further explore this issue in a larger future investigation. Similarly, our observation that carriers of the APOE- 44 allele had elevated $A \beta 42$ levels is interesting, but limited by the small sample size and the nature of the validation cohort.

Our correlation analyses showed that $\mathrm{A} \beta 42$ levels in all brain regions correlated positively with $A \beta 42$ levels in all other brain regions, and that pTau levels in MFG and hippocampus were also positively correlated. Only A $\beta 42$ levels in the neostriatum were weakly correlated with pTau levels in the MFG, presumably reflecting advanced progression of overall AD neuropathologic change associated with neostriatal $A \beta 42$ pathology $[1,2]$. These preliminary findings warrant further investigations with larger study cohorts.
Hence, this highly quantitative, molecularly specific Luminex-based assay will be useful for future studies correlating pathology with genetic and environmental risk factors, clinical and biomarker profiles, and therapeutic interventions. Future applications may specifically include investigation of pathologic changes related to cognitive resilience/reserve to the effects of elevated A $\beta 42$ and pTau levels or to the pathological and phenotypic heterogeneity of AD. Applying this method to specific hippocampal regions, including $\mathrm{CA} 1$ and subiculum, and entorhinal cortex can also help differentiate regional pathology in association with phenotypic outcomes, genetic and environmental risk, and therapeutic interventions.

We envision combining IHC assessments of histopathology in adjacent sections to correlate quantitative findings when needed, and expect this method to complement histologic data sets with standardized and highly quantitative measures of specific markers of neurodegeneration. The combination of a high throughput, low cost approach that uses very little tissue to quantify, in a molecularly specific manner, antigenic targets in banked FFPE tissues will enable diverse studies in AD, but with applications in other diseases of the central nervous system and other organ systems that have large FFPE local or national resources and are not readily accessible through other means.

Acknowledgements We thank Drs. Eric Larson, Thomas Grabowski, and Paul Crane for helpful discussions, Ms. Kimberly Howard for expert histologic support, and Ms. Allison Beller for help with case selection. This work was supported by grants from the NIH (P50 AG005136), Kaiser Permanente Adult Changes in Thought Study (U01 AG006781), and the Nancy and Buster Alvord Endowment.

\section{Compliance with ethical standards}

Conflict of interest The authors declare that they have no conflict of interest.

\section{References}

1. Hyman BT, Phelps $\mathrm{CH}$, Beach TG, et al. National Institute on Aging-Alzheimer's Association guidelines for the neuropathologic assessment of Alzheimer's disease. Alzheimers Dement. 2012;8:1-13

2. Montine TJ, Phelps $\mathrm{CH}$, Beach TG, et al. National Institute on Aging-Alzheimer's Association guidelines for the neuropathologic assessment of Alzheimer's disease: a practical approach. Acta Neuropathol. 2012;123:1-11.

3. Thal DR, Rub U, Orantes M, Braak H. Phases of A betadeposition in the human brain and its relevance for the development of AD. Neurology. 2002;58:1791-800.

4. Braak H, Alafuzoff I, Arzberger T, Kretzschmar H, Del Tredici K. Staging of Alzheimer disease-associated neurofibrillary pathology using paraffin sections and immunocytochemistry. Acta Neuropathol. 2006;112:389-404.

5. Braak H, Braak E. Neuropathological stageing of Alzheimerrelated changes. Acta Neuropathol. 1991;82:239-59. 
6. Mirra SS, Heyman A, McKeel D, et al. The Consortium to Establish a Registry for Alzheimer's Disease (CERAD). Part II. Standardization of the neuropathologic assessment of Alzheimer's disease. Neurology. 1991;41:479-86.

7. Golub VM, Brewer J, Wu X, et al. Neurostereology protocol for unbiased quantification of neuronal injury and neurodegeneration. Front Aging Neurosci. 2015;7:196.

8. Tapias V, Greenamyre JT, Watkins SC. Automated imaging system for fast quantitation of neurons, cell morphology and neurite morphometry in vivo and in vitro. Neurobiol Dis. 2013;54:158-68

9. Walker L, McAleese KE, Johnson M, et al. Quantitative neuropathology: an update on automated methodologies and implications for large scale cohorts. J Neural Transm. 2017;124:671-83.

10. Boyle PA, Yu L, Wilson RS, Schneider JA, Bennett DA. Relation of neuropathology with cognitive decline among older persons without dementia. Front Aging Neurosci. 2013;5:50.

11. Flanagan ME, Larson EB, Walker RL, et al. Associations between use of specific analgesics and concentrations of amyloid-beta 42 or phospho-tau in regions of human cerebral cortex. J Alzheimers Dis. 2018;61:653-62.

12. McNeal DW, Brandner DD, Gong X, et al. Unbiased stereological analysis of reactive astrogliosis to estimate age-associated cerebral white matter injury. J Neuropathol Exp Neurol. 2016;75:539-54.

13. Postupna N, Keene CD, Crane PK, et al. Cerebral cortical Abeta42 and PHF-tau in 325 consecutive brain autopsies stratified by diagnosis, location, and APOE. J Neuropathol Exp Neurol. 2015;74:100-9.

14. Postupna N, Rose SE, Bird TD, et al. Novel antibody capture assay for paraffin-embedded tissue detects wide-ranging amyloid beta and paired helical filament-tau accumulation in cognitively normal older adults. Brain Pathol. 2012;22:472-84.

15. Angeloni S, Cordes R, Dunbar S, et al. $x_{M A P}{ }^{\circledR}$ cookbook. A collection of methods and protocols for developing multiplex assays with xMAP Technology. 3rd ed. Luminex Corp.; 2016.

16. Landlinger $\mathrm{C}$, Preuner S, Willinger B, et al. Species-specific identification of a wide range of clinically relevant fungal pathogens by use of Luminex xMAP technology. J Clin Microbiol. 2009;47:1063-73.
17. Toledo JB, Arnold SE, Raible K, et al. Contribution of cerebrovascular disease in autopsy confirmed neurodegenerative disease cases in the National Alzheimer's Coordinating Centre. Brain. 2013;136:2697-706.

18. Mo Y, Stromswold J, Wilson K, et al. A multinational study distinguishing Alzheimer's and healthy patients using cerebrospinal fluid tau/Abeta42 cutoff with concordance to amyloid positron emission tomography imaging. Alzheimers Dement. 2017;6:201-9.

19. Lovheim H, Elgh F, Johansson A, et al. Plasma concentrations of free amyloid beta cannot predict the development of Alzheimer's disease. Alzheimers Dement. 2017;13:778-82.

20. Shi M, Tang L, Toledo JB, et al. Cerebrospinal fluid alphasynuclein contributes to the differential diagnosis of Alzheimer's disease. Alzheimers Dement 2018;14:1052-1062.

21. Lawlor PA, Bland RJ, Das P, et al. Novel rat Alzheimer's disease models based on AAV-mediated gene transfer to selectively increase hippocampal Abeta levels. Mol Neurodegener. 2007;2:11.

22. Lewis PA, Piper S, Baker M, et al. Expression of BRI-amyloid beta peptide fusion proteins: a novel method for specific highlevel expression of amyloid beta peptides. Biochim Biophys Acta. 2001;1537:58-62.

23. Farrer LA, Cupples LA, Haines JL, et al. Effects of age, sex, and ethnicity on the association between apolipoprotein E genotype and Alzheimer disease. A meta-analysis. APOE and Alzheimer Disease Meta Analysis Consortium. JAMA. 1997;278:1349-56.

24. Steinerman JR, Irizarry M, Scarmeas N, et al. Distinct pools of beta-amyloid in Alzheimer disease-affected brain: a clinicopathologic study. Arch Neurol. 2008;65:906-12.

25. McLean CA, Cherny RA, Fraser FW, et al. Soluble pool of Abeta amyloid as a determinant of severity of neurodegeneration in Alzheimer's disease. Ann Neurol. 1999;46:860-6.

26. Mc Donald JM, Savva GM, Brayne C, et al. The presence of sodium dodecyl sulphate-stable Abeta dimers is strongly associated with Alzheimer-type dementia. Brain. 2010;133:1328-41.

27. Kuo YM, Emmerling MR, Vigo-Pelfrey C, et al. Water-soluble Abeta $(\mathrm{N}-40, \mathrm{~N}-42)$ oligomers in normal and Alzheimer disease brains. J Biol Chem. 1996;271:4077-81. 